\title{
Studying the reducing potencies of antioxidants with the electrochemistry inherently present in electrospray ionization-mass spectrometry
}

\author{
Sabine Plattner • Robert Erb • Jean-Pierre Chervet • \\ Herbert Oberacher
}

Received: 23 July 2013 /Revised: 14 October 2013 / Accepted: 16 October 2013 / Published online: 8 November 2013

(C) The Author(s) 2013. This article is published with open access at Springerlink.com

\begin{abstract}
In this proof-of-principle study, the applicability of electrospray ionization-mass spectrometry (ESI-MS) to characterize the reducing potencies of natural antioxidants is demonstrated. The ESI source represents a controlled-current electrochemical cell. The interfacial potential at the emitter electrode will be at or near the electrochemical potential of those reactions that sufficiently supply all the required current for the ESI circuit. Indicator molecules prone to oxidation in ESI such as amodiaquine were used to visualize the impact of reducing compounds on the interfacial potential. The extent of inhibition of the oxidation of the indicator molecule was found to be dependent on the kind and amount of antioxidant added. Concentration-inhibition curves were constructed and used to compare reducing potencies and to rank antioxidants. This ranking was found to be dependent on the electrode materialindicator molecule combination applied. For fast and automated characterization of the reducing potencies of electrochemically active molecules, a flow-injection system was combined with ESI-MS. Liquid chromatography was used to process complex biological samples, such as red and white wine. Due to their high content of different polyphenols, red wine fractions were found to exhibit higher reducing potencies than the corresponding white wine fractions. Furthermore, for 14 important natural antioxidants, the results obtained with the controlled-current EC-ESI-MS assay were compared to those obtained with chemical antioxidant assays. Irrespectively of the kind of assay used to test the reducing potency, gallic acid, quercetin, and epicatechin were found to
\end{abstract}

S. Plattner $\cdot$ R. Erb $\cdot$ H. Oberacher $(\bowtie)$

Institute of Legal Medicine and Core Facility Metabolomics, Innsbruck Medical University, Müllerstraße 44, 6020 Innsbruck, Austria

e-mail: herbert.oberacher@i-med.ac.at

J.-P. Chervet

Antec, Industrieweg 12, 2382 NV Zouterwoude, The Netherlands be potent reductants. Other antioxidants performed well in one particular assay only. This observation suggests that different kinds of redox and antioxidant chemistry were assessed with each of the assays applied. Therefore, several assays should be used to comprehensively study antioxidants and their reducing potencies.

Keywords Electrospray ionization · Mass spectrometry · Electrochemistry $\cdot$ Oxidation $\cdot$ Antioxidant

\section{Introduction}

Reactive oxygen species (ROS) and reactive nitrogen species (RNS) are the main sources of oxidative stress in biological material. These species can react with lipids, proteins, and nucleic acids giving rise to damage at various sites within the cell. To avoid damage, the cell contains various enzymes and antioxidants (AOXs) to provide protection. AOXs are a complex and diverse group of molecules, and they may be defined as "a compound that when present at relatively low concentrations, compared with those of the oxidisable substrate, significantly delays or inhibits oxidation of that substrate" [1]. Epidemiological evidence suggests that AOXs may be responsible for recognized and potential health benefits of fruits and vegetables in the diet [2].

Factors that provide distinct challenges in studying oxidative stress are that there are multiple oxidant and antioxidant sources, and all compounds have different chemical and physical properties. Based on their mechanism of action, AOXs can be divided into two classes: primary AOXs and secondary AOXs [3, 4]. Primary AOXs actively inhibit oxidation reaction. They can (sacrificially) scavenge ROS/RNS or can inhibit the formation of reactive oxidants [5]. Secondary AOXs inhibit oxidation indirectly by mechanisms such as oxygen-scavenging, light absorption, 
metal chelating, and binding of pro-oxidants. Individual AOXs may act by multiple mechanisms in a single system or may respond in a different manner to different radical and antioxidant sources $[6,7]$. The reducing potency of a compound is one important parameter reflecting one aspect of its antioxidant property. It is oversimplified, however, to refer the reducing potency as antioxidant capacity [5].

Different terms are common to describe different aspects of compound-specific antioxidant properties [4]. Activity deals with the kinetics of a reaction between an AOX and the prooxidant or radical it scavenges or reduces. Capacity measures the thermodynamic conversion efficiency of an oxidant upon reaction with an antioxidant. A number of different chemical assays have been developed for characterizing AOXs, and these have been reviewed [3-5, 7-9]. On the basis of the chemical reactions involved, the assays can be roughly divided into hydrogen atom transfer (HAT) reaction-based assays and single electron transfer (ET) reaction-based assays. In some cases, however, these reaction mechanisms may simultaneously occur. Commonly applied antioxidant assays include the ferric reducing antioxidant power (FRAP) assay [10], the trolox-equivalent antioxidant capacity (TEAC) assay [11], and the 2,2-diphenyl-1-picrylhydrazyl (DPPH) assay [12].

Electrochemical approaches are considered to complement chemical assays in identifying and characterizing AOXs [13, 14]. Electrochemistry (EC) is particularly useful to characterize the reducing potency of a compound, and controlled-potential techniques, such as cyclic voltammetry and amperometry, are commonly applied. Without the use of any reactive species, EC can be considered as a direct test of the physical-chemical properties of an analyte. Conceptually, the oxidation potential and the corresponding current are correlated to a sample's reducing capacity. EC mainly involves electron transfer reactions [15]. Accordingly, EC results might have some correlation with ET assays, but this is still controversially discussed [13]. A clear advantage of EC is that it can readily be combined with chromatographic separation techniques enabling the fast and efficient characterization of individual compounds within complex samples [16]. Moreover, EC techniques can be combined with mass spectrometry (MS) to simultaneously obtain information on reducing potency and molecular structure [17].

Electrospray ionization (ESI)-MS represents a versatile analytical tool for the characterization of small to large molecules and their complexes [18]. EC is an integral part of the ESI process [19-23]. From the electrochemical point of view, the ESI source represents a controlled-current cell consisting of two electrodes [24]. One electrode is the capillary emitter; the mass spectrometer acts as the counter electrode. The electrochemical reactions at the metal-solution interface of the capillary emitter may involve compounds dissolved in the solvent [22, 25, 26], the solvent itself [27], and the electrode material $[28,29]$. The interfacial potential will be at or near the electrochemical potential of those reactions that sufficiently supply all the required current for the ESI circuit.

For the sake of completeness, we want to note that redox reactions in ESI may also occur in the gas phase [30]. Such oxidation reactions are less common than redox reactions in the bulk solution. They typically involve the production of hydroxyl radicals and other reactive oxygen species by corona discharge in the ion source region.

We have recently shown that unwanted electrochemical oxidation of analytes in ESI-MS can efficiently be suppressed by redox buffers (i.e., ascorbic acid, glutathione, and hydroquinone) which are sacrificially oxidized in the ESI emitter [26]. The degree of inhibition was dependent on the kind and concentration of the AOX. Extending this recent communication, we demonstrate herein that the EC of ESI can be used to characterize the reducing potencies of AOXs. Indicator molecules prone to oxidation in ESI such as amodiaquine are used to visualize the impact of AOXs (=redox buffers) on the interfacial potential in the ESI emitter. Concentration-inhibition curves are constructed and used to compare reducing potencies and to rank AOXs. The impact of experimental conditions, such as the electrode material and the kind of indicator molecule used, on the reducing potencies observed is studied. The results of the controlled-current ECESI-MS assay are compared to those obtained with controlledpotential EC-ESI-MS and chemical AOX assays. Finally, the applicability of the developed assay for the characterization of polyphenols in wine samples is demonstrated.

\section{Materials and methods}

\section{Chemicals}

2,2'-Azino-bis-(3-ethylbenzothiazoline-6-sulfonic acid) diammonium salt (ABTS), 2,2-diphenyl-1-picrylhydrazyl (DPPH) radical, peroxidase from horseradish (HRP), 2,4,6tris(2-pyridyl)-s-triazine (TPTZ), iron(III)chloride hexahydrate $\left(\mathrm{FeCl}_{3} \cdot \mathrm{H}_{2} \mathrm{O}\right)$, retinol, $\alpha$-tocopherol, 6-hydroxy2,5,7,8-tetramethylchromane-2-carboxylic acid (trolox), ascorbic acid, hydroquinone, curcumin (from Curcuma longa), melatonin, $\alpha$-lipoic acid, glutathione, ferulic acid, epicatechin, genistein, quercetin, gallic acid, amodiaquine dihydrochloride dihydrate, acetonitrile, methanol, water, and acetic acid were obtained from Sigma Aldrich (St. Louis, MO, USA). Morphine was purchased from Cerilliant (Round Rock, TX, USA). Hydrogen peroxide solution (30\%, $\mathrm{H}_{2} \mathrm{O}_{2}$ ), ammonium hydroxide solution $(25 \%)$, and formic acid were obtained from Fluka (Buchs, Switzerland). Wine samples (Colombard Sauvignon, and Cabernet Syrah; J.P. 
Chenet, Les Grands Chais de France, Petersbach, France) were obtained from a local supermarket.

Flow injection (FI)-ESI-MS for characterizing the reducing potency of AOXs

FI experiments were performed on a Alexys HPLC system (Antec, Zoeterwoude, The Netherlands) online hyphenated to a quadrupole-quadrupole-time-of-flight mass spectrometer (QqTOF; QSTAR XL, AB Sciex, Foster City, CA, USA; Fig. 1a) [31, 32]. The injection volume was $2 \mu$ l. The flow rate was set to $15 \mu \mathrm{l} / \mathrm{min}$. A $10 \mathrm{mM}$ ammonium formate solution ( $\mathrm{pH} 7.3$ ) containing $50 \%$ acetonitrile (v/v) was used as solvent. The Alexys system was controlled by the Clarity software (DataApex, Prague, Czech Republic).

MS was performed in positive ion mode. A modified TurboIonSpray source was used as ESI emitter (Fig. 1b). The spray voltage was applied to a planar electrode device provided by Antec [26, 33]. Electrodes made of conductive diamond, glassy carbon, or platinum $(\mathrm{Pt})$ were available. If not stated otherwise, conductive diamond electrodes were used. The accessible electrode area was approximately $15 \mathrm{~mm}^{2}$. The electrode and the inlet block were separated by a $50-\mu \mathrm{m}$ spacer giving a cell volume of approximately $750 \mathrm{nl}$. Mass spectrometric parameters were optimized using a $5 \mu \mathrm{M}$ solution of amodiaquine in $10 \mathrm{mM}$ ammonium formate $(\mathrm{pH} 7.3)$ containing $50 \%$ acetonitrile $(\mathrm{v} / \mathrm{v})$. The ion spray voltage was set to $4.5 \mathrm{kV}$. Gas flows of 2 (nebulizing gas) and 9 (turbo gas) arbitrary units were employed. The temperature of the turbo gas was adjusted to $200{ }^{\circ} \mathrm{C}$. Mass spectra were acquired from $\mathrm{m} / \mathrm{z}$ 100 to 800 using an accumulation time of $1.0 \mathrm{~s}$. Mass spectra were recorded on a personal computer with the Analyst QS software (version 1.0, service pack 8; AB Sciex).

For characterizing the reducing capacity of an AOX, solutions of the AOX $(0-1 \mathrm{mM})$ were mixed with an indicator compound (amodiaquine, $20 \mu \mathrm{M}$; morphine, $20 \mu \mathrm{M}$; melatonine, $20 \mu \mathrm{M}$ ) and analyzed in triplicate. Peak areas of the oxidized and reduced forms of the indicator molecule were determined in extracted ion chromatograms. Peak area ratios were used to characterize the extent of inhibition of oxidation. To enable a ranking of AOX-specific reducing capacities, the concentration necessary for $50 \%$ inhibition of oxidation of the indicator molecule $\left[\mathrm{IC}_{50}\right.$ (indicator molecule)] was determined.

Screening wine samples for AOXs

A generic solid-phase extraction (SPE) method was used to prepare $4 \mathrm{ml}$ of red and white wine, respectively, for liquid chromatography (LC) fractionation. SPE was accomplished as described elsewhere [34,35]. We only changed the solvents to elute (methanol, $3 \mathrm{ml}$ ) and to reconstitute the analytes extracted (water, $150 \mu \mathrm{l}$ ).
Fig. 1 Schematic diagrams of the flow-injection analysis system (a) and the ESI emitter (b)
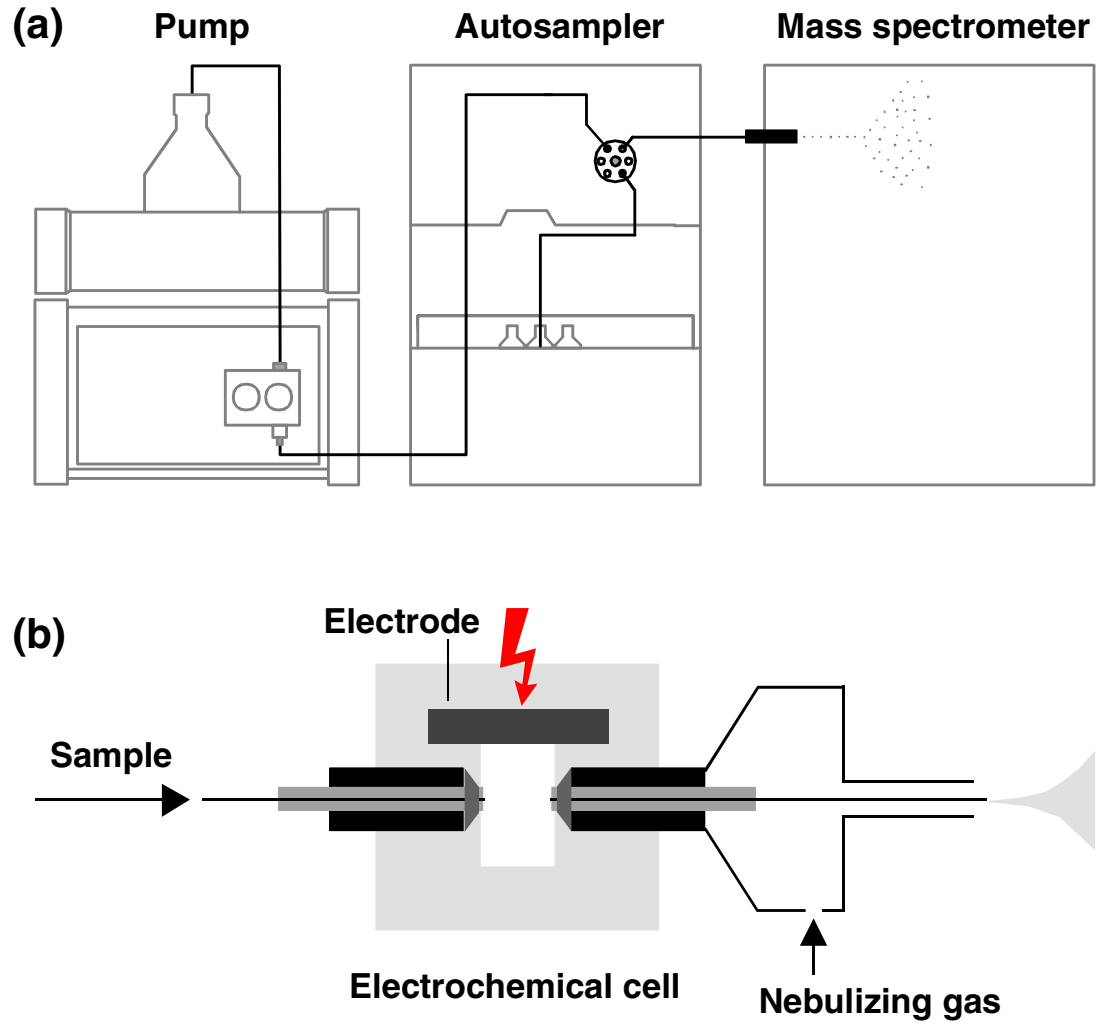
LC fractionation was accomplished on the Alexys HPLC system (Antec). Ten microliters of eluate was injected onto the chromatographic column (Gemini 3u C18, 100×1 mm inner diameter; Phenomenex, Torrance, CA, USA). Chromatographic separations were accomplished with linear gradients of $9-59 \%$ acetonitrile in $0.05 \%$ acetic acid within $17 \mathrm{~min}$. The flow rate was set to $60 \mu \mathrm{l} / \mathrm{min}$ and $15-\mu \mathrm{l}$ fractions were collected. Each fraction was diluted with $15 \mu \mathrm{l}$ of a $20-\mu \mathrm{M}$ solution of amodiaquine in $10 \mathrm{mM}$ ammonium formate $(\mathrm{pH} 7.3)$ and $1 \mu \mathrm{l}$ of $0.5 \%$ ammonium hydroxide solution. The samples were characterized by FI-ESI-MS as described above.

For identification of polyphenols in the wine samples, LCtandem mass spectrometry (MS/MS) under data-dependent acquisition control and automated database search was used [35]. For that purpose, the Alexys HPLC system (Antec) was online hyphenated to the mass spectrometer (QSTAR XL, AB Sciex) $[31,32]$. LC separations of $15-\mu 1$ aliquots of the wine extracts were accomplished as described above. The mass spectrometer was equipped with the TurbolonSpray source. Fused silica capillaries were used as sprayer capillary $(90 \mu \mathrm{m}$ o.d., $20 \mu$ m i.d.; Polymicro Technologies, Phoenix, AZ, USA) and transfer line $(375 \mu \mathrm{m}$ o.d., $20 \mu \mathrm{m}$ i.d.; Polymicro Technologies). Mass spectrometric parameters were optimized using a mixture of quercetin, epicatechin, myricetin, and resveratrol (50 $\mu \mathrm{M}$ each) in $10 \mathrm{mM}$ ammonium formate ( $\mathrm{pH} 7.3$ ) containing $50 \%$ acetonitrile (v/v). Ion spray voltage was set to $4.5 \mathrm{kV}$. Gas flows of 8 (nebulizing gas) and 15 (turbo gas) arbitrary units were employed. The mass spectrometer was operated under data-dependent acquisition control. A duty cycle in the data-dependent acquisition mode included a single MS scan followed by MS/MS scans on the three most abundant precursor ions (processed in reverse order of abundance) not subject to pre-determined or dynamic exclusion. The intensity threshold for triggering MS/MS experiments was set to 50 counts. Isolation of precursor ions was accomplished with Q1 set to unit resolution. A collision gas flow $\left(\mathrm{N}_{2}\right)$ of 5 arbitrary units was used. For each precursor, MS/MS spectra were acquired at three different collision energies $(20,30$, and $40 \mathrm{eV})$. Accumulation times were set to $1.0 \mathrm{~s}$ for MS scans and to $0.5 \mathrm{~s}$ for MS/MS scans. Mass spectra were acquired from $\mathrm{m} / \mathrm{z} 50$ to 700 and recorded on a personal computer with the Analyst QS software (1.0, service pack 8; AB Sciex). Automated library search in the "Wiley Registry of Tandem Mass Spectral Data" was used for compound identification [34-38].

FI-EC-ESI-MS for acquiring mass voltammograms of AOXs

Mass voltammograms of the antioxidants were acquired using the ROXY EC/LC system (Antec) online hyphenated to the mass spectrometer (QSTAR XL; AB Sciex) [31, 32]. The
ROXY system was controlled by the Clarity software (DataApex).

Electrochemical reactions were performed in an electrochemical thin layer cell (ReactorCell; Antec). The reactor cell consisted of a three-electrode arrangement including a working electrode, counter electrode, and a reference electrode. As working electrode, a conductive diamond electrode (Magic Diamond; Antec) was used. The accessible area of the working electrode was $15 \mathrm{~mm}^{2}$. The inlet block of the cell was employed as counter electrode and the HyREF (Antec Leyden) electrode was used as reference electrode. The working electrode and the counter electrode inlet block were separated by a $50-\mu \mathrm{m}$ spacer giving a cell volume of approximately $750 \mathrm{nl}$. Potentials $(0-1,250 \mathrm{mV})$ were applied using a purposive potentiostat (ROXY Potentiostat; Antec). The reactor cell was integrated into the autosampler system by placing it between the injection capillary and the injection valve. Solutions of the AOXs (2$100 \mu \mathrm{M}$ ) were delivered through the electrochemical cell to the $2-\mu 1$ injection loop at a flow rate of $4 \mu \mathrm{l} / \mathrm{min}$.

The mass spectrometer was equipped with the TurboIonSpray source. Fused silica capillaries were used as sprayer capillary $(90 \mu \mathrm{m}$ o.d., $20 \mu \mathrm{m}$ i.d.; Polymicro Technologies) and transfer line (375 $\mu \mathrm{m}$ o.d., $20 \mu \mathrm{m}$ i.d.; Polymicro Technologies). The flow rate was set to $3 \mu 1 / \mathrm{min}$. The remaining mass spectrometric parameters were as described above.

\section{Chemical AOX assays}

The following chemical assays were used to characterize the antioxidant capacities of the tested AOXs: the FRAP assay [10], the TEAC assay [11], and the DPPH assay [12]. All measurements were performed in triplicate on an 8452A diode array spectrophotometer (Hewlett-Packard, Palo Alto, CA, USA).

To prepare the ABTS radical solution for the TEAC assay, $7 \mu \mathrm{l}$ of a $10 \mathrm{mg} / \mathrm{ml}$ aqueous solution of ABTS, $3.2 \mu \mathrm{l}$ of $0.06 \%$ aqueous solution of $\mathrm{H}_{2} \mathrm{O}_{2}$, and $33 \mu \mathrm{l}$ of a $1 \mathrm{mg} / \mathrm{ml}$ aqueous solution of HRP were diluted with $25 \mathrm{mM}$ ammonium formate buffer $(\mathrm{pH} 7.3)$ to a final volume of $1 \mathrm{ml}$. Antioxidant capacity was evaluated by adding different amounts of AOX $(10 \mu \mathrm{l}$, final concentration $2.5-50 \mu \mathrm{M})$ to the ABTS solution. After 10 min of reaction, the absorbance decrease at $730 \mathrm{~nm}$ was measured. To enable a ranking of AOX-specific antioxidant capacities, the AOX concentration necessary to induce a $50 \%$ decrease of absorbance $\left[\mathrm{IC}_{50}(\mathrm{TEAC})\right]$ was determined.

For characterizing antioxidant capacity with the DPPH assay, a methanolic solution of the DPPH radical $(100 \mu \mathrm{M}$, $1 \mathrm{ml}$ ) was mixed with different concentrations of the AOXs $(10 \mu \mathrm{l}$, final concentration $2.5-50 \mu \mathrm{M})$. Ten minutes after addition of the AOX solution, the absorbance decrease at 
$515 \mathrm{~nm}$ was measured. To enable a ranking of AOX-specific antioxidant capacities, the concentration necessary to induce a $50 \%$ decrease of absorbance $\left[\mathrm{IC}_{50}(\mathrm{DPPH})\right]$ was determined.

For characterizing antioxidant capacity with the FRAP assay, TPTZ $(1.25 \mathrm{ml}, 10 \mathrm{mM}$ in $40 \mathrm{mM} \mathrm{HCl}), 12.5 \mathrm{ml}$ of ammonium acetate buffer ( $\mathrm{pH} 3.6)$, and $1.25 \mathrm{ml}$ of $\mathrm{FeCl}_{3} \cdot \mathrm{H}_{2} \mathrm{O}$ $(20 \mathrm{mM})$ were mixed. Next, different amounts of AOX $(20 \mu \mathrm{l}$, final concentration $2.5-100 \mu \mathrm{M}$ ) were added to $2 \mathrm{ml}$ of the freshly prepared FRAP reagent. After $5 \mathrm{~min}$ of reaction at $37^{\circ} \mathrm{C}$, the change of absorbance at $593 \mathrm{~nm}$ was measured. To enable a ranking of AOX-specific antioxidant capacities, the concentration necessary to reach $50 \%$ of the maximum absorption $\left[\left(\mathrm{IC}_{50}(\mathrm{FRAP})\right]\right.$ was determined.

\section{Results and discussion}

Assessing the reducing potency of AOXs with ESI-MS

EC is a competent approach to study the reducing potency of AOXs. Usually, potentiostatic techniques are employed, where the current is measured as function of the applied electrochemical potential. Herein, we present an alternative approach. The EC of ESI is used to study AOXs. From the electrochemical point of view [23], the ESI source represents a controlled-current cell, and the interfacial potential at the emitter electrode will be at or near the electrochemical potential of electrochemical reactions that sufficiently supply all the required current for the ESI circuit. Therefore, the dynamically adjusted potential would represent the readout for characterizing the compound-specific reducing potency. This value, however, cannot be directly measured without modification of the emitter setup. Accordingly, we decided to indirectly characterize the redox chemistry at the emitter electrode. The idea was to use an indicator compound for that purpose. An appropriate indicator compound should be prone to oxidation in ESI, and two forms should be detected by MS, a reduced and an oxidized form. The addition of a redoxactive compound should induce a decrease of the electrochemical potential, which should further give rise to a shift of signal intensity from the oxidized form of the indicator compound to its reduced form. The observed extent of oxidation inhibition would be governed by the redox chemistry of the AOX and the amount of AOX added.

For selecting an appropriate indicator compound, the following criteria were defined: (1) the compound should be prone to oxidation in ESI, (2) the redox chemistry should be simple (only one dominant oxidation reaction should occur), (3) the compound and its oxidation product should be detectable by ESI-MS, (4) the compound should be synthetic, and (5) the reagent costs should be low.

One compound that met the criteria was the drug molecule amodiaquine (Amo). Amo is prone to oxidation in positive
ESI. The redox chemistry of Amo is shown in Scheme 1. Amo is converted by a two-electron oxidation reaction into the quinoneimine form (Amo-2H). Amo and Amo-2H are detectable by ESI-MS (Fig. 2a).

To demonstrate the effect of AOX addition on Amo oxidation, ascorbic acid was selected as test compound. Ascorbic acid is used on a wide scale as an antioxidant agent in foods and drinks; therefore, it represents an important quality indicator of foodstuffs. Furthermore, ascorbic acid is important for therapeutic purposes and is involved in metabolic processes. Due to its reductive properties, ascorbic acid represents an efficient homogenous redox buffer for ESI [26]. The effect of mixing different amounts of ascorbic acid with Amo on the extent of Amo oxidation is shown in Fig. 2. As expected, the ratio of Amo-2H to Amo changed in the presence of different amounts of AOX (Fig. 2). Fifty micromolar of ascorbic acid halved the amount of Amo-2H produced; $250 \mu \mathrm{M}$ of ascorbic acid completely inhibited the formation of Amo- $2 \mathrm{H}$.

For fast and automated characterization of the reducing potencies of AOXs, a FI-ESI-MS system was developed (Fig. 1a). To calculate the extent of inhibition of Amo oxidation, the peak areas of Amo and Amo-2H, respectively, in the corresponding extracted ion chromatograms were used. With the FI-ESI-MS system, the reducing properties of 14 important natural AOXs were characterized (Table 1). For each AOX, inhibition of Amo oxidation as function of AOX concentration in the sample solution was determined. Representative concentration-inhibition curves are shown in Fig. 3. Quercetin (Que) was found to inhibit oxidation at concentrations above $50 \mu \mathrm{M}$. Maximum inhibition was obtained at $0.5 \mathrm{mM}$. The concentration necessary for $50 \%$ inhibition of Amo oxidation [ $\left.\mathrm{IC}_{50}(\mathrm{Amo})\right]$ was found to be $160 \mu$ M. $\alpha$-Tocopherol (Toc) addition had hardly any effect on Amo oxidation. Obviously, Toc is less effectively oxidized at the emitter electrode than Amo. Genistein (Gen) seems to represent a pro-oxidant. Increasing amounts of Gen increased the extent of Amo oxidation. The $\mathrm{IC}_{50}$ (Amo) values determined for all 14 tested AOXs are summarized in Table 1. Gen, Toc, and retinol showed no or a very low reducing potency. For ferulic acid (Fer), hydroquinone (Hyd), melatonin (Mel), and $\alpha$-lipoic acid (Lip), $50 \%$ inhibition of Amo oxidation was not reached at $1.0 \mathrm{mM}$. Thus, $\mathrm{IC}_{50}(\mathrm{Amo})$ values were estimated by extrapolation. For all other AOXs, $\mathrm{IC}_{50}(\mathrm{Amo})$ values were directly deduced from concentration-inhibition curves.

Dependence of reducing potency on the electrode material and the indicator compound used

As part of assay development and characterization, the impact of the electrode material on the $\mathrm{IC}_{50}(\mathrm{Amo})$ values was evaluated. The 14 AOXs were used as test samples. The electrode materials compared included conductive diamond, 
Scheme 1 Amodiaquine structure and oxidation pathway

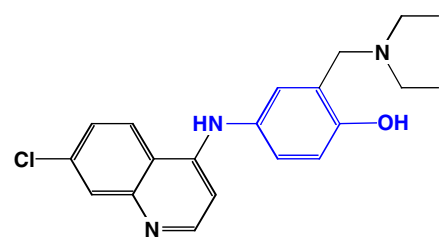

Amo

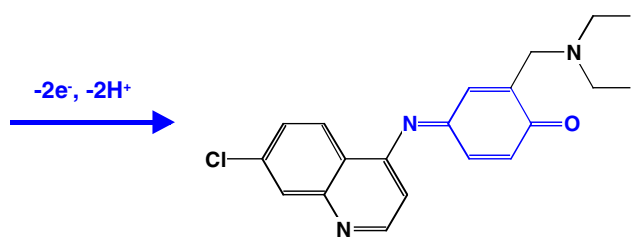

Amo-2H glassy carbon, and Pt. In Fig. 4, the results obtained for seven AOXs are summarized; the $\mathrm{IC}_{50}$ (Amo) values of these seven compounds were directly deduced from concentrationinhibition curves. There was a good correlation between the $\mathrm{IC}_{50}$ (Amo) values measured using the carbon-based

\section{(a) $10 \mu \mathrm{M}$ amodiaquine}

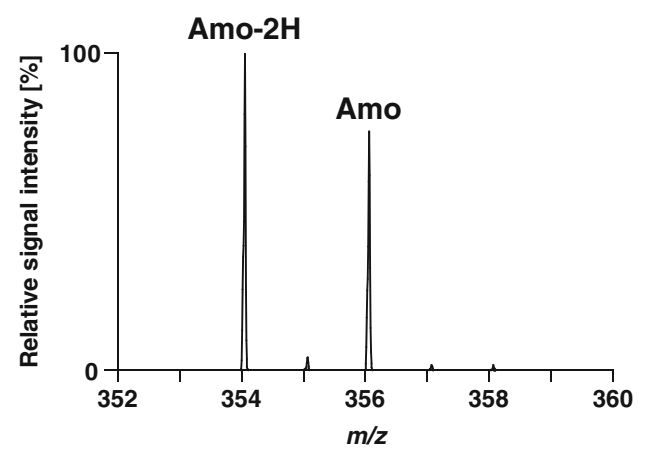

(b) $10 \mu \mathrm{M}$ amodiaquine $+50 \mu \mathrm{M}$ ascorbic acid

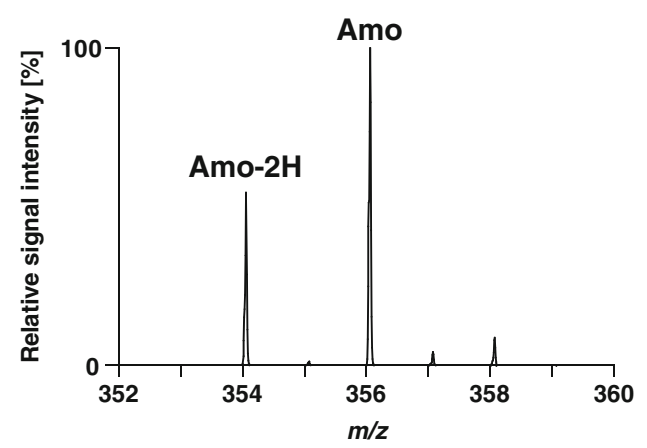

(c) $10 \mu \mathrm{M}$ amodiaquine $+250 \mu \mathrm{M}$ ascorbic acid

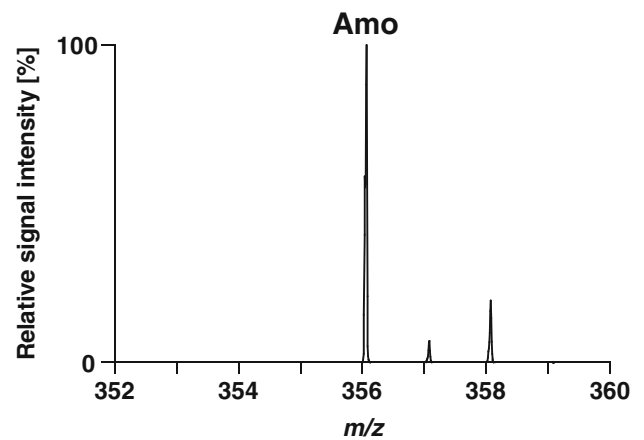

Fig. 2 Mass spectra of sample solutions containing $10 \mu \mathrm{M}$ amodiaquine and $0 \mu \mathrm{M}$ ascorbic acid (a), $50 \mu \mathrm{M}$ ascorbic acid (b), and $250 \mu \mathrm{M}$ ascorbic acid (c), respectively electrodes $\left(R^{2}=0.88\right)$. On these electrodes, the relative ranking of the AOXs was found to be almost identical. Only ascorbic acid (Asc) and gallic acid (Gal) changed positions. Interestingly, for all compounds the $\mathrm{IC}_{50}$ (Amo) values measured with the Pt electrode were lower than the values obtained with the carbon-based electrodes. Furthermore, several AOXs changed their positions in the relative ranking compared to the carbon-based electrodes rankings. These observations clearly suggest that the reducing potency of an AOX observed is dependent on the electrode material used.

In a further set of experiments, the impact of the indicator molecule chemistry on the relative ranking of the AOXs in terms of reducing potency was studied (Table 2). The indicator molecules tested included Amo, morphine, and melatonine. Oxidation of the targets was accomplished on the conductive diamond electrode. Depending on the kind of indicator molecule used, hydroxylation, dimerization, or dehydration reactions were observed. With each setup, concentrationinhibition curves were acquired for each of the 14 AOXs tested. The $\mathrm{IC}_{50}$ values obtained were used to rank the AOXs. Parts of these rankings are summarized in Table 2. Comparison of the rankings clearly revealed that the reducing potency of an AOX observed is dependent on the chemistry of the indicator molecule used.

The compound-specific reducing potency determined by ESI-MS is influenced by the experimental setup used. The measured $\mathrm{IC}_{50}$ value is at least dependent on the electrochemical properties of the AOX, the electrode material, and the indicator molecule. Probably, comparing the results from multiple experiments would be necessary to fully characterize the reducing potencies of AOXs and to rank them. Further studies will be necessary to identify a minimum set of indicator molecule-electrode material combinations appropriate for comprehensive testing.

With all different kinds of EC-ESI-MS setups tested, Gal was found to be among the three most efficient AOXs. This observation clearly suggests that Gal is a redox buffer efficiently working in diverse electrochemical environments.

Comparing controlled-current and controlled-potential ECESI-MS for characterizing the reducing potencies of AOXs

Controlled-potential EC can be combined with MS to characterize compound-specific redox properties. The 
Table 1 Summary of experimental results obtained by studying the reducing potencies of 14 important natural AOXs with different assays

\begin{tabular}{|c|c|c|c|c|c|c|}
\hline Compound & Compound class & $\mathrm{IC}_{50}(\mathrm{Amo})(\mu \mathrm{M})$ & $E_{1 / 2}(\mathrm{mV})$ & $\mathrm{IC}_{50}(\mathrm{DPPH})(\mu \mathrm{M})$ & $\mathrm{IC}_{50}(\mathrm{TEAC})(\mu \mathrm{M})$ & $\mathrm{IC}_{50}$ (FRAP) $(\mu \mathrm{M})$ \\
\hline Ascorbic acid (Asc) & Vitamin & 50 & 850 & 27 & 24 & 41 \\
\hline Curcumin (Cur) & Polyphenol & 860 & 660 & 24 & 10 & 72 \\
\hline Epicatechin (Epi) & Flavanol & 500 & 970 & 8 & 7 & 37 \\
\hline Ferulic acid (Fer) & Phenolic acid & $1,550^{\mathrm{c}}$ & 800 & 39 & 6 & 41 \\
\hline Gallic acid (Gal) & Phenolic acid & 90 & 875 & 7 & 4 & 13 \\
\hline Genistein (Gen) & Isoflavone & n.a. ${ }^{\text {a }}$ & 955 & n.a. & 5 & n.a. \\
\hline Glutathione (Glu) & Peptide & 390 & 985 & 49 & 12 & n.a. \\
\hline Hydroquinone (Hyd) & Phenol & $1350^{\mathrm{c}}$ & n.d. ${ }^{b}$ & 27 & 18 & 34 \\
\hline Melatonin (Mel) & Hormone & $1,400^{\mathrm{c}}$ & 560 & n.a. & 6 & n.a. \\
\hline Quercetin (Que) & Flavonol & 160 & 780 & 8 & 5 & 27 \\
\hline Retinol (Ret) & Vitamin & n.a. & n.d. & n.a. & 40 & n.a. \\
\hline Trolox (Tro) & Vitamin derivative & 520 & 830 & 28 & 29 & 37 \\
\hline$\alpha$-Lipoic acid (Lip) & Coenzyme & $1,400^{\mathrm{c}}$ & 975 & n.a. & n.a. & n.a. \\
\hline$\alpha$-Tocopherol (Toc) & Vitamin & n.a. & n.d. & 28 & 34 & 29 \\
\hline
\end{tabular}

${ }^{\mathrm{a}}$ n.a. no or very low activity

${ }^{\mathrm{b}}$ n.d. not determined

${ }^{\mathrm{c}}$ Extrapolated values

obtained mass voltammogram provides qualitative information about the redox reaction studied; the half-wave potential $\left(E_{1 / 2}\right)$ is closely related to the standard potential.

Mass voltammograms were obtained for 11 out of the 14 AOXs studied. Three compounds were not detectable by ESIMS in positive ion mode. Thus, no mass voltammogram was acquired for these compounds. The $E_{1 / 2}$ values obtained for the remaining compounds are summarized in Table 1. There was hardly any correlation between the $E_{1 / 2}$ values and the corresponding $\mathrm{IC}_{50}(\mathrm{Amo})$ values. The discrepancy between

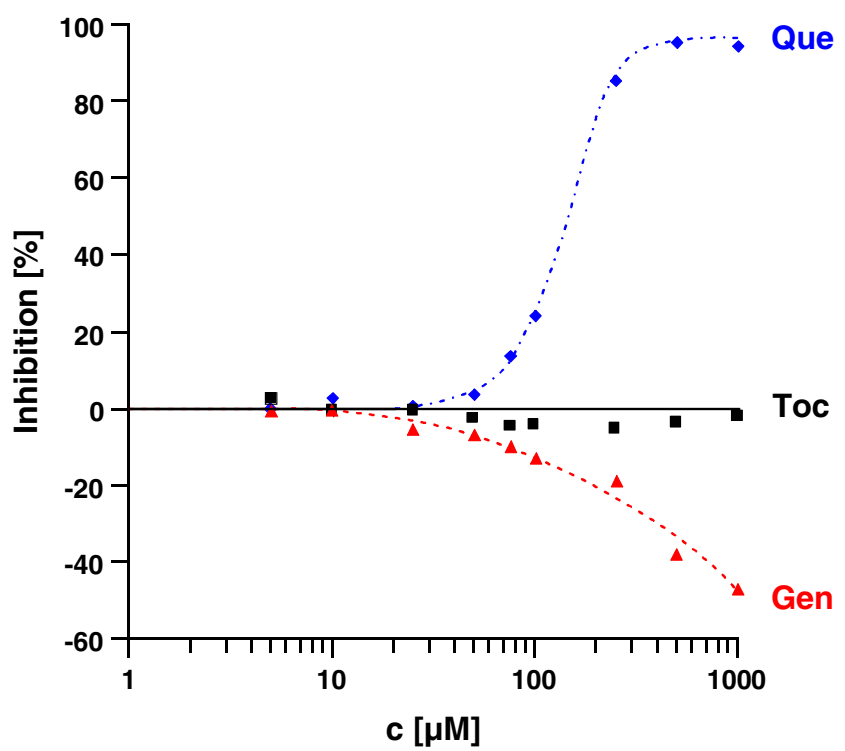

Fig. 3 Concentration-inhibition curves for assessing the reducing potencies of quercetin, $\alpha$-tocopherol, and genistein controlled-current and controlled-potential EC-ESI-MS results can to some extent be explained by differences in assay designs. In controlled-potential EC-ESI-MS, the redox chemistry of the targeted compound is studied only. It is well known, however, that oxidation products show redox activities themselves [17, 39]. The resulting cumulative reducing potency is assessed with the controlled-current EC method, and this parameter is probably more relevant for characterizing an $\mathrm{AOX}$ than $E_{1 / 2}$ of a single redox system only.

Another advantage of the controlled-current EC method is the ability to characterize compounds that are not detected or difficult to detect by ESI-MS in positive ion mode. Due to the use of an indicator molecule, neutral or acidic compounds can be studied as well.

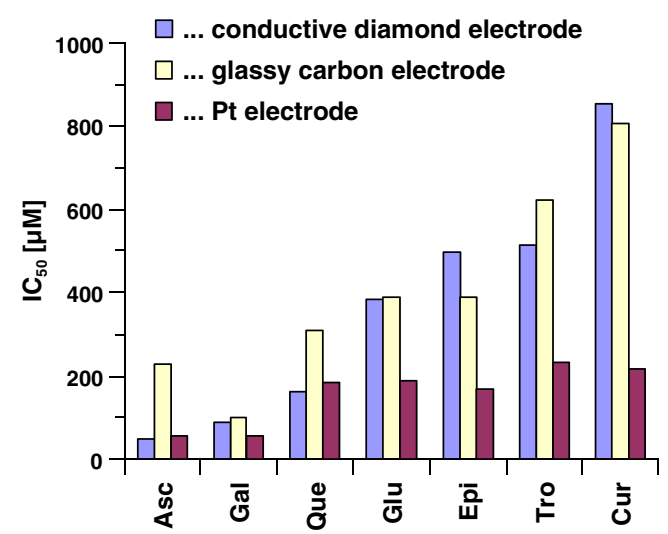

Fig. 4 Comparing the potencies of different antioxidants to suppress amodiaquine oxidation on conductive diamond, glassy carbon, and Pt 
Table 2 Summary of AOXs showing high potencies to suppress the oxidation of different indicator molecules on the conductive diamond electrode

\begin{tabular}{lll}
\hline Indicator compound & Oxidation product & Most efficient antioxidants \\
\hline Amodiaquine & Amodiaquine-2H & Asc $>$ Gal $>$ Que \\
Morphine & Morphine $+\mathrm{O}$ & $\mathrm{Glu}>\mathrm{Gal}>\mathrm{Que}$ \\
Melatonine & $(\text { Melatonine })_{2}$ & $\mathrm{Epi}>\mathrm{Gal}>\mathrm{Asc}$ \\
\hline
\end{tabular}

Comparing the controlled-current EC-ESI-MS assay with chemical AOX assays

For method development and validation, reference methods are needed to demonstrate the relevance of measured data. For the characterization of AOX, a number of assays are accepted $[3,4]$. Currently, however, there is no single standardized reference assay available to compare AOXs. Usually, several different assays are applied to comprehensively study the different aspects of AOX activity, and often the results obtained with these assays show limited comparability.

EC mainly involves electron transfer reactions [15]. Accordingly, EC results might have some correlation with ET-based chemical AOX assays [13]. To test this hypothesis, the set of 14 AOXs was characterized with commonly applied antioxidant assays, including the FRAP assay [10], the TEAC assay [11], and the DPPH assay [12]. With each assay, $\mathrm{IC}_{50}$ values were determined to enable direct comparison. Scatter diagrams are depicted in Fig. 5. The $\mathrm{IC}_{50}$ values measured with different assays showed no or only weak linear correlations. Also, among the chemical assays, inconsistencies were observed. Some AOXs performed well in one particular assay only. For Asc, a very low $\mathrm{IC}_{50}(\mathrm{Amo})$ value was obtained. The high reducing potency of Asc, however, was not confirmed by any of the chemical assays. Ferulic acid (Fer) was found to be a potent AOX in the TEAC assay only; Hyd in the FRAP assay only.

Thermodynamic and kinetic effects may have influenced the $\mathrm{IC}_{50}$ values measured. There were major differences between the assays with respect to the kind of oxidant used. Radicals were applied in the TEAC and the DPPH assays. A ferric salt is the oxidant in the FRAP assay. In the EC-ESI-MS assay, oxidation occurs on an electrode. Furthermore, reaction times differed significantly between the chemical assays and the electrochemical assay. The change of absorbance was measured 5-10 min after the reaction started; the residence time in the electrochemical cell was approximately $3 \mathrm{~s}$ only.

Despite lack of linear correlation between the $\mathrm{IC}_{50}$ values obtained with different assays, there was noticeable agreement among the assays with respect to ranking of the most potent AOXs. Particularly three compounds were located in the lower left corners of almost all the scatter diagrams generated (Fig. 5), which indicated high potencies in the assays compared. Gal was found to be the most effective AOX. Gal (a)

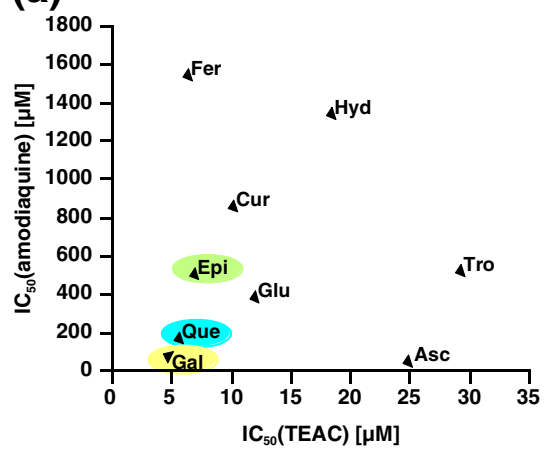

(d)

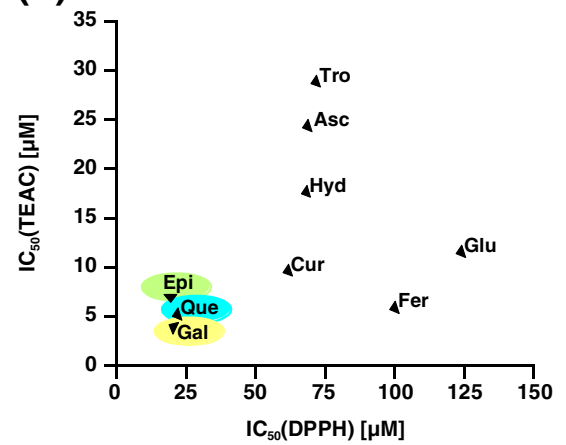

(b)

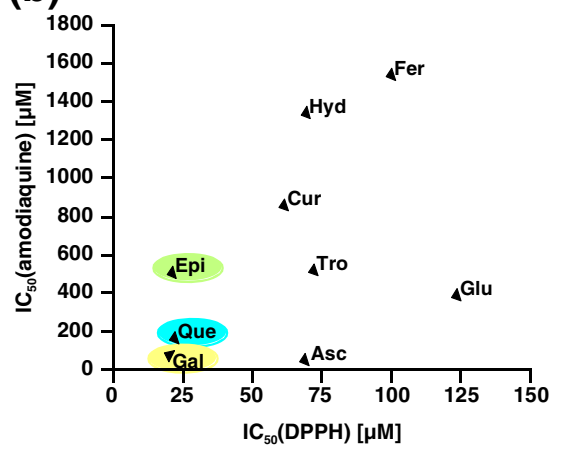

(e)

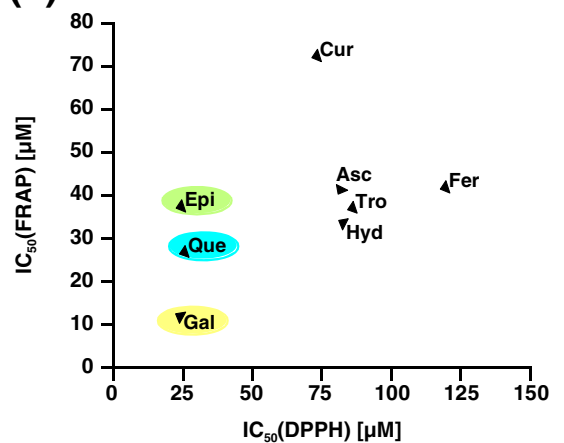

(c)

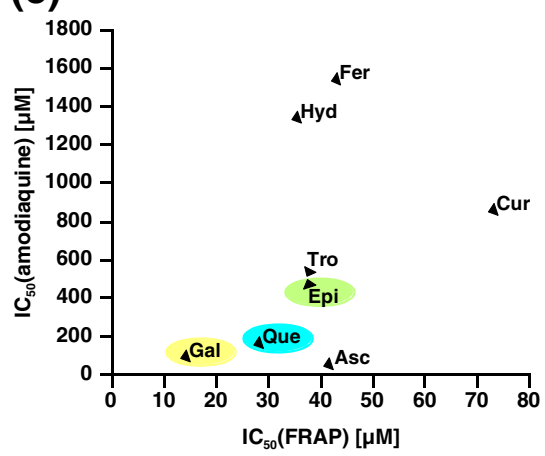

(f)

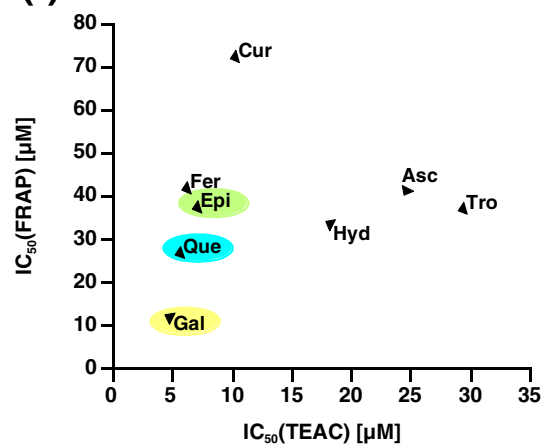

Fig. 5 Scatter diagrams to compare the $\mathrm{IC}_{50}$ values of natural antioxidants measured with the TEAC assay, the DPPH assay, the FRAP assay, and controlled-current EC-ESI-MS using amodiaquine as indicator molecule. Gallic acid, quercetin, and epicatechin are highlighted 
Fig. 6 Comparison of peak areas (total, Amo, Amo-2H) obtained from the analysis of red wine and white wine fractions with controlled-current EC-ESI-MS using amodiaquine as indicator molecule (a) Red wine
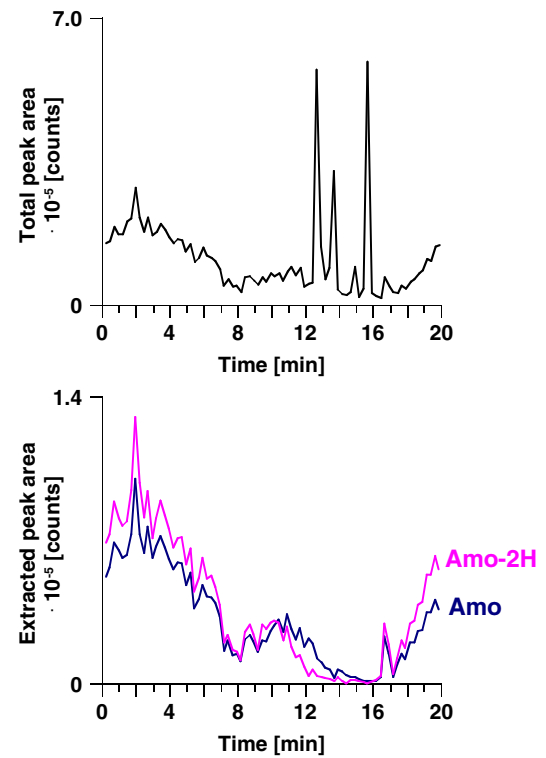

(b) White wine
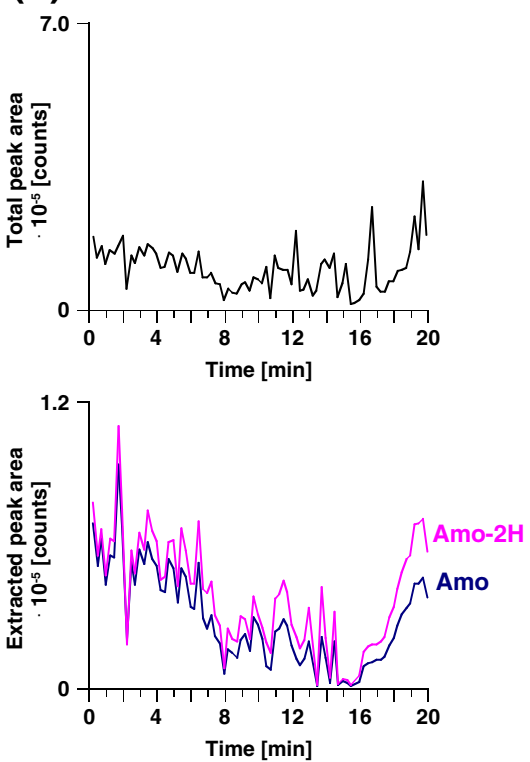

is a naturally occurring plant phenol that was found to be a strong AOX. Gal is used in processed food, cosmetics, and food packing materials to prevent rancidity induced by lipid peroxidation and spoilage. The next best AOXs were quercetin (Que) and epicatechin (Epi). These polyphenols are found in many fruits, vegetables, leaves, and grains.
Screening wine samples for AOXs

To challenge the developed electrochemical assay, experiments were carried out using biological samples, in this case, wine samples. Wine is a source of polyphenols. Many of these compounds (e.g., resveratrol and quercetin) are known
Fig. 7 Comparison of the reducing potencies of chromatographic fractions of a red wine sample, a white wine sample, and a blank sample determined with controlledcurrent EC-ESI-MS using amodiaquine as indicator molecule

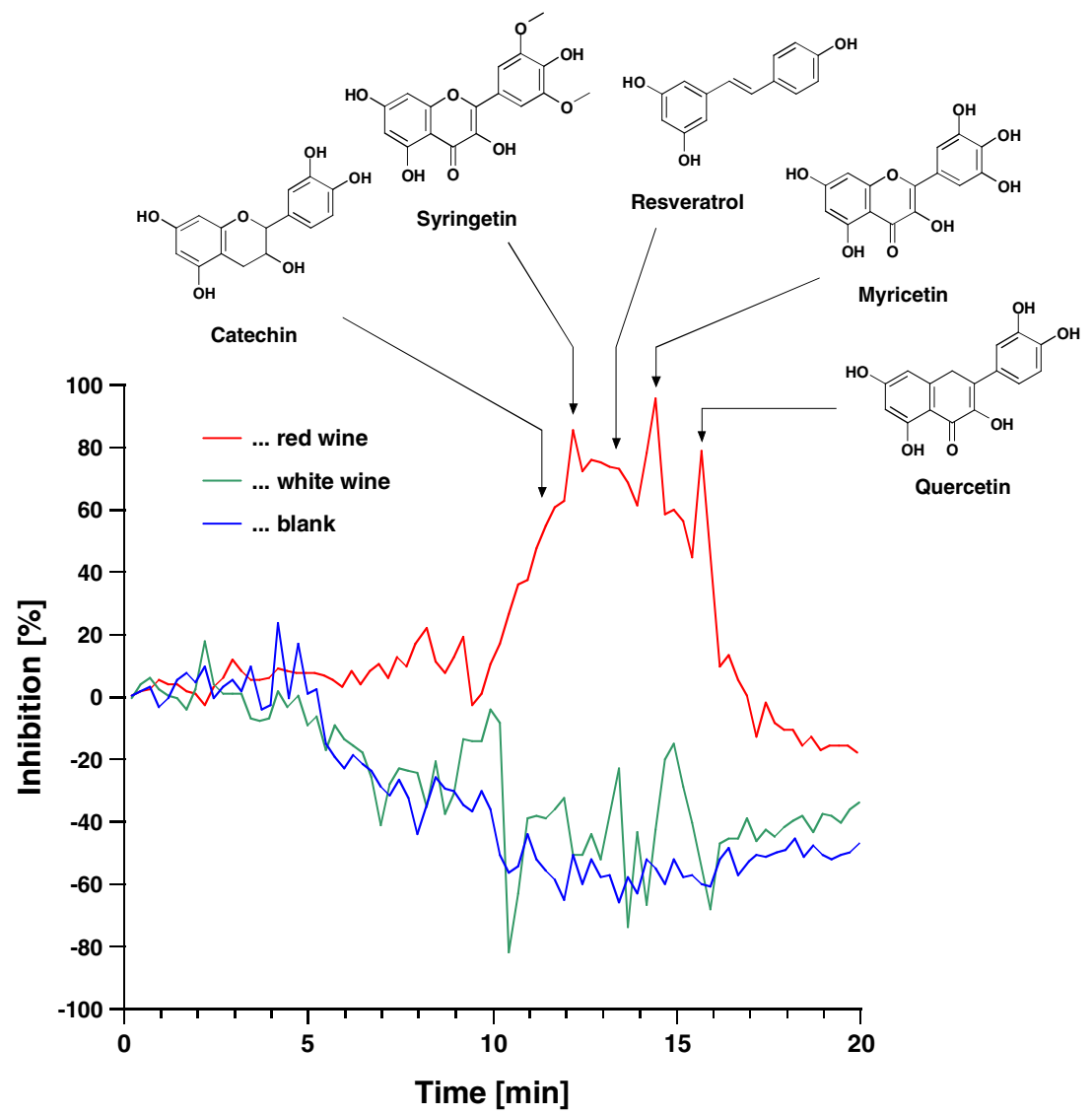


(a) Catechin

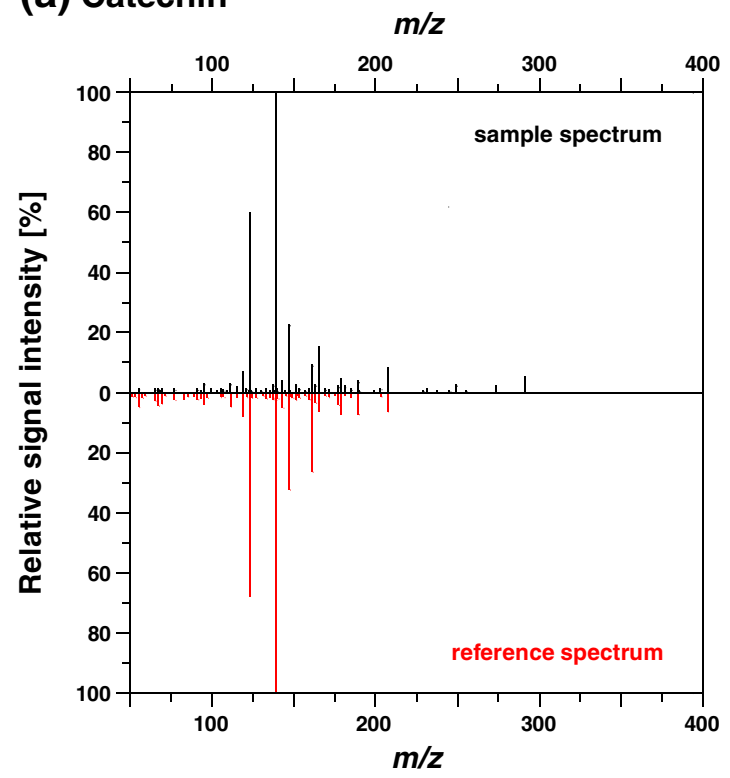

(c) Myricetin

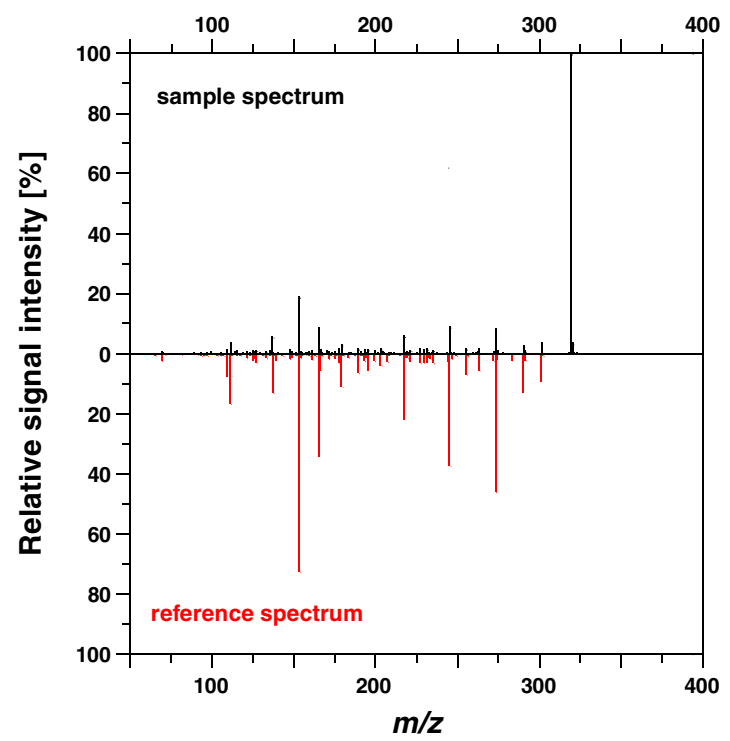

(b) Resveratrol

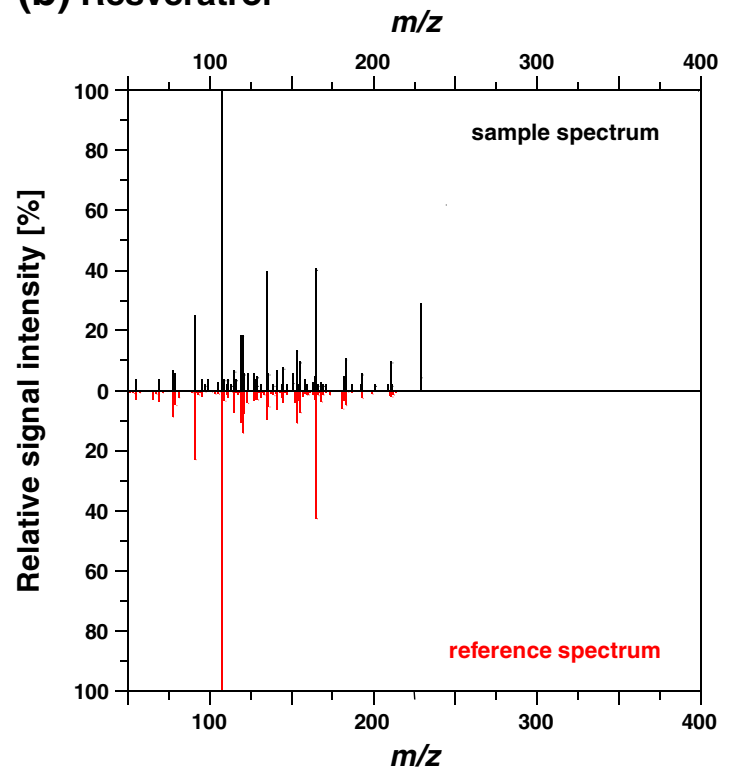

(d) Quercetin

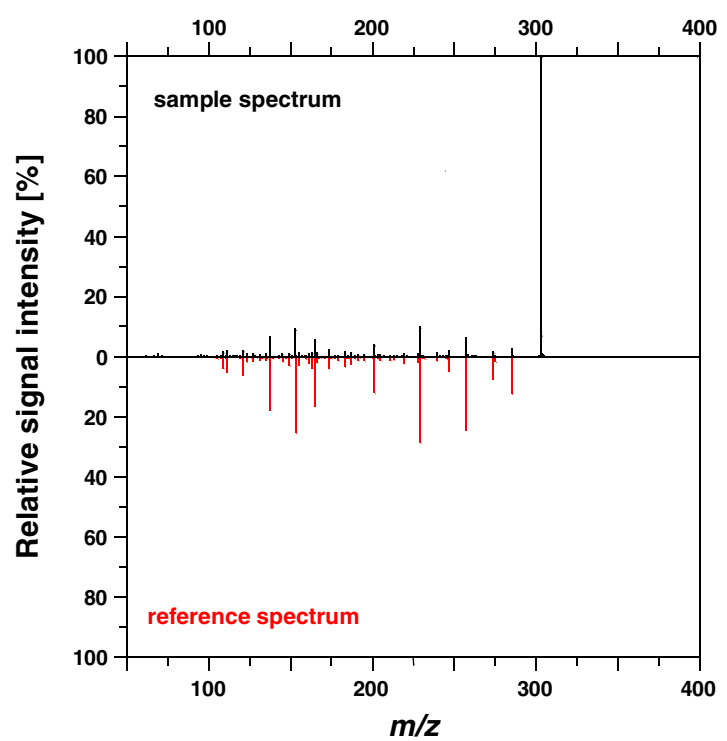

Fig. 8 Tandem mass spectra of catechin, resveratrol, myricetin, and quercetin from a red wine sample in comparison to reference spectra

for their antioxidant potency. Typically, a higher content of polyphenols can be found in red wine compared to white wine [40]. Accordingly, red wine samples usually show higher AOX potencies than white wine samples [41].

The controlled-current EC-ESI-MS assay was applied to characterize the reducing potencies of fractions of red and white wine. Two samples of commercial wines from France were bought in a nearby supermarket and were prepared for FI-ESI-MS with SPE and consecutive LC separation. Each fraction collected represented a retention time window of $15 \mathrm{~s}$ in the LC separation. The fraction-specific peak areas obtained by FI-ESI-MS were used to construct total ion current chromatograms as well as Amo- and Amo-2H-specific ion current chromatograms (Fig. 6). Comparison of the total ion current chromatograms clearly revealed that the red wine sample contained a higher content of detectable polyphenols than the white wine sample. In fractions of both wine samples, ion suppression was observed. The occurrence of ion suppression effects was indicated by the reduction of indicator molecule-specific signal intensities. Ion suppression represents a severe problem for ESI-MS-based quantification. In the context of AOX screening, however, we believe that ion suppression can be very much tolerated. As the oxidized and the reduced forms of Amo should have very similar ionization efficiencies, ion suppression will only decrease the total intensities without significantly changing the signal intensity 
ratio used to characterize the reducing potency of a specific fraction.

For each fraction collected, the degree of inhibition of Amo oxidation was determined. Obtained time-inhibition diagrams are shown in Fig. 7. For each wine sample, the ratio of Amo$2 \mathrm{H}$ to Amo observed in the first fraction was used to set the base value of $0 \%$ inhibition. Furthermore, a blank sample was processed to define the baseline. As indicated by a negative drift of this baseline, variation of the organic content during gradient elution changed the rate of Amo oxidation. As expected, red wine fractions showed a higher degree of inhibition than white wine fractions, which can be explained by differences in polyphenol content. Highest degrees of inhibition were observed for the red wine fractions collected between 10 and $16.5 \mathrm{~min}$. For the fraction collected at $14.4 \mathrm{~min}$, more than $95 \%$ inhibition of Amo oxidation was observed, and myrecitin was identified as the polyphenol responsible for the reducing potency indicated. Other polyphenols identified that effectively suppressed Amo oxidation included catechin, syringetin, resveratrol, and quercetin (Fig. 8).

\section{Conclusions}

The concept of homogenous redox buffers to suppress oxidation in ESI is well known. Redox buffering works by decreasing the interfacial potential at the ESI emitter. Accordingly, efficient redox buffers have low redox potentials and high reducing potency, and can therefore efficiently suppress the oxidation of other compounds in the ESI emitter. The extent of inhibition is dependent on the kind and concentration of the redox buffer.

AOXs may act by scavenging ROS/RNS or by inhibiting the formation of reactive oxidants. Thus, AOXs may posses reducing potency, and the EC of ESI is an appropriate tool to characterize that parameter. The decrease of the interfacial potential at the emitter electrode is indicated by the inhibition of the oxidation of an indicator molecule. Appropriate indicator molecules should be prone to oxidation in ESI, and the reduced and the oxidized form should be detectable by MS. Amodiaquine, reserpine, morphine, and melatonine were shown to represent suitable indicator molecules.

$\mathrm{IC}_{50}$ values can be determined from concentration-inhibition curves. These values can be used to compare and rank AOXs. To some extent, the electrochemical properties of the indicator molecule and the electrode material influence the compoundspecific reducing potencies observed, which suggests that multiple experimental conditions should be used to comprehensively characterize the reducing potency of an AOX.

The oxidation of AOX will lead to products that show redox activities themselves. Accordingly, the standard potential of the oxidation of an AOX will not reflect the full reducing potency of an AOX provided. The controlled-current EC method was shown to assess such a cumulative reducing potency.

Automated and fast characterization of AOXs can be accomplished by using FI-ESI-MS. Furthermore, for the screening of complex biological samples, the assay can be combined with LC separation.

Electrochemical reactions are mainly electron transferbased reactions. It was, therefore, hypothesized that EC results might have some correlation with ET-based AOX assays. Our experiments revealed that there is no or only a weak linear correlation between the $\mathrm{IC}_{50}$ values determined by the different (electro)chemical assays. Thermodynamic and kinetic effects may be responsible for the observed differences. Each individual assay characterizes different kinds of redox and AOX chemistry, and this observation clearly suggests that several assays should be used to comprehensively study the different aspects of AOXs.

Despite lack of linear correlation between $\mathrm{IC}_{50}$ values obtained with different assays, there was noticeable agreement among the chemical and electrochemical assays with respect to ranking of the most potent AOXs. Gallic acid was found to be most effective AOX. The next best AOXs were quercetin and epicatechin. These polyphenols can be found in many fruits, vegetables, leaves, and grains.

Acknowledgment This work was funded by the Austrian Science Fund (FWF): P 22526-B11.

Open Access This article is distributed under the terms of the Creative Commons Attribution License which permits any use, distribution, and reproduction in any medium, provided the original author(s) and the source are credited.

\section{References}

1. Gutteridge JMC (1994) Rev Clin Gerontol 4:279-288

2. Halvorsen BL, Carlsen MH, Phillips KM, Bohn SK, Holte K, Jacobs DR Jr, Blomhoff R (2006) Am J Clin Nutr 84:95-135

3. Craft BD, Kerrihard AL, Amarowicz R, Pegg RB (2012) Compr Rev Food Sci Food Saf 11:148-173

4. Apak R, Gorinstein S, Böhm V, Schaich KM, Özyürek M, Güclü K (2013) Pure Appl Chem 85:957-998

5. Huang D, Ou B, Prior RL (2005) J Agric Food Chem 53:1841-1856

6. Ishige K, Schubert D, Sagara Y (2001) Free Radic Biol Med 30:433-446

7. Prior RL, Wu X, Schaich K (2005) J Agric Food Chem 53:4290 4302

8. Frankel EN, Finley JW (2008) J Agric Food Chem 56:4901-4908

9. Pinchuk I, Shoval H, Dotan Y, Lichtenberg D (2012) Chem Phys Lipids 165:638-647

10. Benzie IF, Strain JJ (1996) Anal Biochem 239:70-76

11. Miller NJ, Rice-Evans C, Davies MJ, Gopinathan V, Milner A (1993) Clin Sci 84:407-412

12. Brand-Williams W, Cuvelier ME, Berset C (1995) Lebensm Wiss Technol 28:25-30

13. Blasco AJ, Gonzalez Crevillen A, Gonzalez MC, Escarpa A (2007) Electroanalysis 19:2275-2286

14. Escarpa A (2012) Chem Rec 12:72-91 
15. Jurva U, Wikstrom HV, Weidolf L, Bruins AP (2003) Rapid Commun Mass Spectrom 17:800-810

16. Niederlander HA, van Beek TA, Bartasiute A, Koleva II (2008) J Chromatogr A 1210:121-134

17. Zettersten C, Co M, Wende S, Turner C, Nyholm L, Sjoberg PJ (2009) Anal Chem 81:8968-8977

18. Yamashita M, Fenn JB (1984) J Chem Phys 88:4451-4459

19. Blades AT, Ikonomou MG, Kebarle P (1991) Anal Chem 63:2109_ 2114

20. Van Berkel GJ, McLuckey SA, Glish GL (1991) Anal Chem 63: 1098-1109

21. Van Berkel GJ, Kertesz V (2007) Anal Chem 79:5510-5520

22. Van Berkel GJ, McLuckey SA, Glish GL (1992) Anal Chem 64: 1586-1593

23. Jackson GS, Enke CG (1999) Anal Chem 71:3777-3784

24. Van Berkel GJ, Zhou F (1995) Anal Chem 67:2916-2923

25. Van Berkel GJ, Kertesz V, Ford MJ, Granger MC (2004) J Am Soc Mass Spectrom 15:1755-1766

26. Plattner S, Erb R, Chervet JP, Oberacher H (2012) Anal Bioanal Chem 404:1571-1579

27. Van Berkel GJ, Zhou F, Aronson JT (1997) Int J Mass Spectrom 162: $55-67$

28. Van Berkel GJ, Kertesz V (2001) J Mass Spectrom 36:1125-1132
29. Peintler-Krivan E, Van Berkel GJ, Kertesz V (2010) Rapid Commun Mass Spectrom 24:1327-1334

30. Boys BL, Kuprowski MC, Noel JJ, Konermann L (2009) Anal Chem 81:4027-4034

31. Erb R, Plattner S, Pitterl F, Brouwer HJ, Oberacher H (2012) Electrophoresis 33:614-621

32. Plattner S, Erb R, Pitterl F, Brouwer HJ, Oberacher H (2012) J Chromatogr B 883-884:198-204

33. Van Berkel GJ, Asano KG, Kertesz V (2002) Anal Chem 74:5047-5056

34. Schubert B, Oberacher H (2011) J Chromatogr A 1218:3413-3422

35. Oberacher H, Schubert B, Libiseller K, Schweissgut A (2013) Anal Chim Acta 770:121-131

36. Oberacher H (2011) Wiley Registry of Tandem Mass Spectral Data, MSforID. Wiley, Hoboken

37. Oberacher H, Pavlic M, Libiseller K, Schubert B, Sulyok M, Schuhmacher R, Csaszar E, Kofeler HC (2009) J Mass Spectrom 44:494-502

38. Pavlic M, Schubert B, Libiseller K, Oberacher H (2010) Forensic Sci Int 197:40-47

39. Arts MJTJ, Haenen GRMM, Voss H-P, Bast A (2004) Food Chem Toxicol 42:45-49

40. Vinson JA, Hontz BA (1995) J Agric Food Chem 43:401-403

41. Fernandez-Pachon MS, Villano D, Garcia-Parrilla MC, Troncoso AM (2004) Anal Chim Acta 513:113-118 